\title{
Self-stabilizing Numerical Iterative Computation
}

\author{
Ezra N. Hoch, Danny Bickson and Danny Dolev \\ School of Computer Science and Engineering \\ The Hebrew University of Jerusalem \\ Jerusalem, Israel
}

July 10, 2008

\begin{abstract}
Many challenging tasks in sensor networks, including sensor calibration, ranking of nodes, monitoring, event region detection, collaborative filtering, collaborative signal processing, etc. can be formulated as a problem of solving a linear system of equations. Several recent works propose different distributed algorithms for solving to these problems, usually by linear iterative numerical methods.

In this work, we extend the settings of the above approaches, by adding another dimension to the problem. Specifically, we are interested in self-stabilizing algorithms, that continuously run and converge to a solution from any initial starting state. This aspect of the problem is highly important because of the dynamic nature of the network and the frequent changes in the measured environment.

In this paper, we link together algorithms from two different domains. On the one hand, we use the rich linear algebra literature of linear iterative methods for solving systems of linear equations, which are naturally distributed with rapid convergence properties. On the other hand, we are interested in self-stabilizing algorithms, where the input to the computation is constantly changing, and we would like the algorithms to converge from any initial state. We propose a simple novel method called SS-ITERATIVE as a self-stabilizing variant of the linear iterative methods. We prove that under mild conditions the self-stabilizing algorithm converges to a desired result. We further extend these results to handle the asynchronous case.

As a case study, we discuss the sensor calibration problem and provide simulation results to support the applicability of our approach.
\end{abstract}

Keywords: Self-stabilizing, sensor networks, iterative methods, numerical methods, sensor calibration, Jacobi Algorithm.

Contact author: Ezra Hoch

Email: ezra.hoch@gmail.com

Tel: $+972-523-961-865$

Regular Submission

Entitled for Best Student Paper Award. 


\section{Introduction}

Consider a distributed system of sensors measuring real-world data. Sensors are located in different areas; for example, the senors are spread throughout a building and they measure the temperature to adjust the heating or cooling. We would like the collected data to be as reliable as possible, reflecting closely the changing environmental conditions. One of the obstacles we face when designing algorithms which collect data from a sensor network are measurement errors. There are two main types of inaccuracies of sensors' measurements: noisy environment and sensing equipment which is not calibrated. It is desirable that sensors could execute a distributed algorithm for calibrating their environmental readings. In this setting sensors are allowed to communicate among themselves, using data from other nodes to affect their reported individual reading. Furthermore, we would like our calibration algorithm to have fault-tolerance properties. Specifically, we are interested in self-stabilizing algorithms [7] which converge to an optimal solution from any initial state.

In this work we combine techniques from two different domains. On the one hand, our solution relies heavily on previous research in the field of numerical iterative methods. Following [10], [12],[9] and many other related works, we show that the calibration of local sensors' readings can be formulated as a linear system of equations $A \mathbf{x}=\mathbf{b}$, where $\mathbf{x}$ represents the output reading, $\mathbf{b}$ represents the local reading, and $A$ represents a weighted communication graph. This formulation allows us to utilize the vast results in the field of linear algebra. On the other hand, we would like our desired algorithm to have self-stabilizing properties.

The main challenge we have faced in this work, is that in the classical linear algebra literature $\mathbf{b}$ is assumed to be constant. In our settings, the environment is constantly changing and the computed algorithm never terminates, leading to constantly changing values of $\mathbf{b}$. In this paper, we ask the following question: "Is it possible to devise a self-stabilizing numerical iterative method?" We answer affirmatively, and show that under minor conditions it is possible to devise a self-stabilizing algorithm that solves a dynamic system of linear equations, where the input to the system is constantly changing.

To the best of our knowledge, this is the first work tackling this challenging problem. We believe that our approach can have numerous applications in the field of distributed self-stabilizing computation. Among these potential applications are distributed ranking algorithms of nodes and data items [3], collaborative filtering [1], localization [10], collaborative signal processing [12], region detection [9], etc.

Other works discuss fault tolerance aspects of the distributed computation. For example, overcoming faults in sensors by averaging the input was investigated in [11] providing a centralized algorithm. Quantifying faulty nodes' effect on the system's output is discussed in [8] and [5]. These papers consider bounded input paths and their effect on the stability of the output. In [6] infinite input paths are considered under the assumption that only specific sensors' input may change. All three papers consider discrete input values, as opposed to a continuous set of input values discussed in this paper.

The paper is constructed as follows. Section 2 defines the model and problem definition. Section 3 presents our novel algorithm SS-Iterative. Section 4 analyzes our algorithm and gives bounds on the convergence rate. Section 5 presents experimental results of running SS-ITERATIVE using sample topologies. Section 6 extends our construction the asynchronous case. We conclude in Section 7. 


\section{Model and Problem Definition}

We model the sensor calibration problem as follows. Given a directed communication graph $G=$ $(V, E), V$ is the set of nodes $V=\left\{p_{1}, \ldots, p_{n}\right\}, E$ is the set of weighted edges connecting them (weights can be negative). Edge weights are used to model the bidirectional dependence between nodes' outputs. For each $p_{j} \in N\left(p_{i}\right)$ the weighted edge $w_{p_{i}, p_{j}}$ specifies the linear gain of $p_{i}$ on $p_{j}$. (If $p_{j} \notin N\left(p_{i}\right)$ then $w_{p_{i}, p_{j}}=0$.) In addition, we require a non-zero self connected edge, $w_{p_{i}, p_{i}} \neq 0$, which represents the weight of $p_{i}$ 's own input.

Initially, we assume a synchronous system: during a single round of communication, any pair of connected nodes may send a single message on each directed edge. Each round $r$, each node $p_{i}$ has a scalar input value $I_{p_{i}}(r)$, which represents the local reading of the sensor. ${ }^{1}$ In addition, $p_{i}$ outputs its output value, which is denoted by $O_{p_{i}}(r)$. Denote by $I(r)$ the input vector of the entire system at round $r$, and by $O(r)$ the output vector of the system at the end of round $r$. In Section 6 we relax the assumption of synchronous rounds and provide a variant of the algorithm which works in asynchronous settings.

As an example, consider a network with 3 nodes $p_{1}, p_{2}$ and $p_{3}$ located in an oval room. $p_{1}$ is located in the center of the room, and $p_{2}, p_{3}$ are located in the opposite sides. We would like $p_{1}$ 's reading to be 1.1 times its own value, and 0.2 times each of its neighbors values. That is, $O_{p_{1}}(r)=$ $1.1 \cdot I_{p_{1}}(r)+0.2\left(O_{p_{2}}(r)+O_{p_{2}}(r)\right)$. Thus, we would set $w_{p_{1}, p_{1}}=1.1$ and $w_{p_{1}, p_{2}}=w_{p_{1}, p_{3}}=0.2$.

In the above example, $p_{1}$ 's output depended on $p_{2}$ and $p_{3}$ 's output. If $p_{3}$ 's output also depends on $p_{1}$ output, i.e., $w_{p_{3}, p_{1}} \neq 0$, then there is a "circular dependency" of the output values. This imposes a challenge, since the target is to ensure that in steady state the output vector $O(r)$ satisfies $O(r+1)=O(r)$ while preserving the linear relations among the different nodes' outputs and inputs. We require some definitions to formally state this goal.

The schematic operation of each node $p_{i}$ at round $r$ is composed of the following steps: (a) read the value of $I_{p_{i}}(r)$; (b) send messages; (c) receive messages; and (d) do some processing and output $O_{p_{i}}(r)$. Then a new round is started, and the nodes continue so forever.

Definition 2.1. A configuration $\mathcal{C}$ of the system at round $r$ consists of the state of each node prior to performing any operation at round $r$; this configuration is denoted by $\mathcal{C}(r)$.

The above definition states that the value of the input read at round $r$ is not part of the configuration. In addition, when a node sends a message to other nodes, they may contain information regarding its current input and its previously received messages. Thus, messages sent at round $r$ may not contain information regarding computations done at other nodes during round $r$.

Definition 2.2. An input sequence $\mathcal{I}$ of length $\ell$ is a list of $\ell$ vectors such that each $\mathbf{v} \in \mathcal{I}$ is a possible input vector of the system (i.e., $\mathbf{v} \in \mathfrak{D}$, the domain of allowed values). An output sequence $\mathcal{O}$ of length $\ell$ is a list such that each $\mathbf{v} \in \mathcal{O}$ can potentially be an output vector of the system.

Definition 2.3. A step from configuration $\mathcal{C}$ to configuration $\mathcal{C}^{\prime}$ on input vector $\mathbf{v}$ is legal if $\mathcal{C}^{\prime}$ is reached from $\mathcal{C}$ by the system when having $\mathbf{v}$ as the input vector. $\mathbf{u}$ is produced by a legal step if $\mathbf{u}$ is the output vector of the system resulting from such a legal step.

\footnotetext{
${ }^{1}$ For simplicity of notations we use scalar variables in the paper. An extension to the vector case is immediate.
} 
DEFINITION 2.4. A run of a system on input sequence $\mathcal{I}=\{\mathbf{v}(1), \ldots, \mathbf{v}(\ell)\}$ starting from configuration $\mathcal{C}(r)$ is the sequence $\mathcal{C}(r), O(r), \mathcal{C}(r+1), O(r+1), \ldots$ s.t. the step from $\mathcal{C}(r+i)$ to $\mathcal{C}(r+i+1)$ on input vector $\mathbf{v}(i+1)$ is legal, and $O(r+i)$ is produced by that legal step.

The system is said to produce the output sequence $\mathcal{O}=\{O(r), \ldots, O(r+\ell-1)\}$.

In the special case when the sensor observations (the input to the system) are fixed, the output decision of the sensors should converge to a solution that preserves the linear relations among node inputs and outputs. More formally, consider an input sequence $\mathcal{I}$ of identical input vectors; i.e., $\mathcal{I}=\{\mathbf{v}, \mathbf{v}, \mathbf{v}, \ldots\}$. It is desired that for such an input a run from any configuration $\mathcal{C}$ on $\mathcal{I}$ would end up producing an output sequence $\mathcal{O}=\{\mathbf{u}(1), \mathbf{u}(2), \ldots\}$ such that $\|\mathbf{u}(i)-\mathbf{u}\| \rightarrow 0$ as $i \rightarrow \infty$, for a $\mathbf{u}$ that solves the following linear system of equations:

$$
\mathbf{u}_{i}=w_{p_{i}, p_{i}} \cdot \mathbf{v}_{i}+\sum_{p_{j} \in N\left(p_{i}\right)} w_{p_{i}, p_{j}} \cdot \mathbf{u}_{j} .
$$

We assume that the above equations are uniquely solvable, noting $\mathbf{u}$ is the solution to $\mathbf{v}$.

One of the most efficient distributed approaches for solving a set of linear equations of the type $A \mathbf{x}=\mathbf{b}$ is by using linear iterative algorithms. Unlike Gaussian elimination, which has a cost of $O\left(n^{3}\right)$, where $n$ is the number of variables, an iterative algorithm usually solves a system of linear equations in time of $O\left(n^{2} r\right.$, ) where $r$ is the number of iterations, which is typically logarithmic in $n$. These algorithm are naturally distributed and work well in asynchronous settings. Furthermore, when converging, the algorithms converge to a solution from any initial state. An excellent survey of such methods is found in [2].

Our main novel contribution in this paper is in borrowing algorithms from the linear iterative methods domain and analyzing their self-stabilizing properties. In a practical setting, it is highly unreasonable to assume that sensor readings do not change over time. However, it is reasonable to assume that at steady state the change in sensor readings is bounded. Informally, in this work we show that once the input readings are bounded, the output solution is bounded as well. This useful observation enables us to tie together numerical iterative methods and dynamically changing environments in a self-stabilizing manner.

The following definition bounds the change in sensor observations:

Definition 2.5. An input sequence $\mathcal{I}=\{\mathbf{v}(1), \mathbf{v}(2), \ldots, \mathbf{v}(\ell)\}$ is $\delta$-bounded around $\mathbf{v}$ if for any $1 \leq i \leq \ell$, it holds that $\|\mathbf{v}(i)-\mathbf{v}\|_{\infty} \leq \delta .^{2}$

Definition 2.5 states that a sequence $\mathcal{I}$ is $\delta$-bounded if all the vectors in $\mathcal{I}$ are bounded within an $n$ dimensional cube with an edge $2 \delta$, centered around a point $\mathbf{v}$. We note that once changes in the input are not bounded, then no efficient algorithm (especially in a network that is sparsely connected) can calculate the output fast enough. For example, if the diameter of the communication graph is $\mathcal{D}$, for some system of equations it would take at least $\mathcal{D}$ rounds for the information about readings at one side of the network to propagate to the other side of the network.

DeFinition 2.6. Let $\mathcal{I}$ be an input sequence that is $\delta$-bounded around $\mathbf{v}$ and let $\mathbf{u}$ be the solution to input $\mathbf{v}$. A run from configuration $\mathcal{C}$ on input sequence $\mathcal{I}, \epsilon$-converges to its solution if the produced output sequence $\mathcal{O}=\{\mathbf{u}(1), \mathbf{u}(2), \ldots \mathbf{u}(\Delta t)\}$ satisfies that $\| \mathbf{u}(\Delta t)-\mathbf{u}) \|_{\infty} \leq \epsilon(\Delta t, \delta, \mathcal{C})$; where $\epsilon$ is a function of $\Delta t, \delta$ and $\mathcal{C}$.

$$
{ }^{2}|| x \|_{\infty}=\max \left\{\left|x_{i}\right|\right\} .
$$


Definition 2.6 requires that if - starting from configuration $\mathcal{C}$ - the inputs are in an $n$ dimensional box of radius $\delta$ around $\mathbf{v}$ then the output in time $\Delta t$ is bounded as well within an $n$ dimensional box around $\mathbf{u}$ with radius $\epsilon(\Delta t, \delta, \mathcal{C})$. We aim at an $\epsilon(\Delta t, \delta, \mathcal{C})$ that decreases as $\Delta t$ increases, as long as the inputs are bounded by the same $\mathbf{v}$-centered, $\delta$-radius box. Clearly, for $\delta>0, \epsilon(\Delta t, \delta, \mathcal{C})>0$ for any $\Delta t$. That is, there is some minimal radius $\delta^{\prime}>0$ around $\mathbf{u}$ s.t. we cannot ensure a tighter bound.

The above definition considers a single initial configuration, and a single input sequence $\mathcal{I}$. We are interested in an algorithm that works for all initial configurations and all input sequences.

Definition 2.7. An algorithm $\mathcal{A} \epsilon$-converges for $\delta$-bounded input sequence $\mathcal{I}$ if every run (from any configuration) on $\mathcal{I}, \epsilon$-converges to its solution. An algorithm $\mathcal{A} \epsilon$-always converges if for every $\delta$-bounded input sequence $\mathcal{I}, \mathcal{A} \epsilon$-converges.

Definition 2.7 formally defines the problem at hand, as an algorithm $\mathcal{A}$ that always converges has the desired self-stabilizing property: for any system state, once the sensors' readings changes are bounded, the change in output of the entire system is bounded as well.

Our goal is to find an algorithm $\mathcal{A}$ that is $\epsilon$-always converging for a provably "good" $\epsilon$. Moreover, we aim at having $\mathcal{A}$ efficient also in its message complexity and simplicity of code, allowing lightweight sensors to actually implement it.

\section{Our Proposed Solution}

An equivalent formulation of the update rule Eq. (1) is

$$
\mathbf{u}_{i}=w_{p_{i}, p_{i}} \cdot \mathbf{v}_{i}+\sum_{j \neq i} w_{p_{i}, p_{j}} \cdot \mathbf{u}_{j} .
$$

The above equation states a condition on $p_{i}$ 's output, in regard to $p_{i}$ 's inputs and $p_{i}$ 's neighbors. Thus, it encapsulates the requirement that different nodes influence each other's reported readings, while taking into consideration their local readings as well.

Since $w_{p_{i}, p_{i}} \neq 0$, the above equation can be stated as:

$$
\frac{1}{w_{p_{i}, p_{i}}} \mathbf{u}_{i}-\sum_{j \neq i} \frac{w_{p_{i}, p_{j}}}{w_{p_{i}, p_{i}}} \cdot \mathbf{u}_{j}=\mathbf{v}_{i}
$$

By denoting $w_{i, j}=-\frac{w_{p_{i}, p_{j}}}{w_{p_{i}, p_{i}}}$ and $w_{i, i}=\frac{1}{w_{p_{i}, p_{i}}}$ we get:

$$
\sum_{j} w_{i, j} \cdot \mathbf{u}_{j}=\mathbf{v}_{i}
$$

Let $W$ be the matrix that has $w_{i, j}$ as entries, Eq. (2) can be written in linear algebra notation, (s.t. it applies to all nodes simultaneously):

$$
W \mathbf{u}=\mathbf{v} .
$$

If we consider a non-self-stabilizing system in which the inputs do not change (that is, the input is fixed to $\mathbf{v}$ ), then Eq. (3) can be seen as $A \mathbf{x}=\mathbf{b}$, where $A$ and $\mathbf{b}$ are given. In such a case, we 
are interested in finding the value of $\mathbf{x}$, which is a vector of $n$ unknown variables. However, we are interested in the case where $\mathbf{v}$ changes over time, and thus Eq. (3) does not describe the problem properly, but rather helps in understanding the motivation for our solution.

We use a modified update rule (relative to Eq. (1)):

$$
O_{p_{i}}(r+1)=w_{p_{i}, p_{i}} \cdot I_{p_{i}}(r+1)+\sum_{j \neq i} w_{p_{i}, p_{j}} \cdot O_{p_{j}}(r) .
$$

Clearly, for the case of $\delta=0$, a 0 -bounded input sequence $\mathcal{I}$, if $\left(O_{p_{i}}(r+1)-O_{p_{i}}(r)\right) \longrightarrow 0$ as $r \rightarrow \infty$ then Eq. (4) converges to the solution of Eq. (1). Thus, if the update rule of Eq. (4) is executed simultaneously by all nodes, and for all of the nodes $\left(O_{p_{i}}(r+1)-O_{p_{i}}(r)\right) \longrightarrow 0$, then it also solves Eq. (3). That is, if each node locally executes Eq. (4) then the global solution is reached. This observation motivates algorithm SS-ITERATIVE in Figure 1.

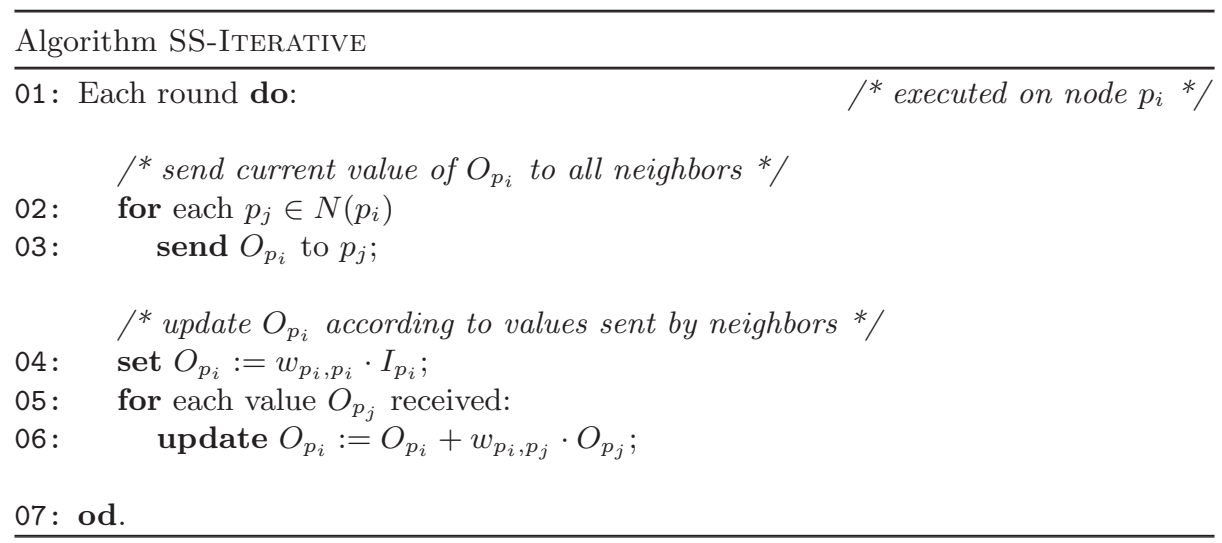

Figure 1: A self-stabilizing iterative algorithm.

REMARK 3.1. In SS-ITERATIVE there is no notion of the "current round number $r$ ". That is, $p_{i}$ reads and writes to the variables $I_{p_{i}}$ and $O_{p_{i}}$ without being "aware" of $r$. When we discuss the algorithm "from the outside", we will consider $I_{p_{i}}(r)$ and $O_{p_{i}}(r)$ instead of just $I_{p_{i}}, O_{p_{i}}$.

Consider $p_{i}$ is running at round $r+1$. When $p_{i}$ performs Line 03, it sends the value of $O_{p_{i}}$. The last time $O_{p_{i}}$ was updated was at Line 04 and Line 06 of round $r$. Thus, the value sent by $p_{i}$ at round $r+1$ is actually $O_{p_{i}}(r)$. Therefore, the values received from $p_{j}$ by $p_{i}$ and used to update $O_{p_{i}}(r+1)$ are $O_{p_{j}}(r)$. However, the value read by $p_{i}$ in Line 04 is the value of $I_{p_{i}}(r+1)$. Concluding that $p_{i}$ updates $O_{p_{i}}(r+1)$ exactly according to Eq. (4).

REMARK 3.2. Each node $p_{i}$ must know the values of $w_{p_{i}, p_{j}}$ as "part of the code". Thus, these values cannot be subject to transient faults.

\section{Analysis of SS-Iterative}

[2] shows that the update rule Eq. (4) can be written in linear algebra form as

$$
O(r+1)=A I(r+1)+B O(r),
$$


where $A$ is a diagonal matrix with $w_{p_{i}, p_{i}}$ in the main diagonal, and $B_{i j}=w_{p_{i}, p_{j}}$ for $i \neq j$

$$
A \triangleq(\operatorname{diag}\{W\})^{-1} \quad, \quad B \triangleq-\left(A W-I_{n \times n}\right)
$$

where $I_{n \times n}$ is the identity matrix.

As noted in Section 2, when the input sequence is constant (i.e., $I(r)=\mathbf{v}$ for all $r$ ) the iterative execution of the above equations converges to $\mathbf{u}=A \mathbf{v}+B \mathbf{u}$, which is the same as $\mathbf{u}=W^{-1} \mathbf{v}$, thus solving Eq. (3). Following, we analyze the result of iteratively applying these equations for $\delta$-bounded input sequences.

Let $\mathcal{I}$ be an input sequence of length $\ell$ that is $\delta$-bounded around vector $\mathbf{v}$. That is, $\mathcal{I}=$ $I(r), I(r+1), \ldots, I(r+\ell-1)$ for some round $r$. Note that SS-ITERATIVE saves a single scalar variable at each node, and thus the configuration of round $r+1$ can be defined by the value of $O(r)$ at round $r$. Consider SS-ITERATIVE's run, starting from an arbitrary configuration at round $r$. We aim at showing that $O(r+\Delta t)$ is bounded by a cube centered at $\mathbf{u}$. Denote by $\mathbf{c}(\Delta t) \triangleq O(r+\Delta t)-\mathbf{u}$. If we show that $\|\mathbf{c}(\Delta t)\|_{\infty}$ is bounded (as $\Delta t$ increases), then $O(r+\Delta t)$ is within a bounded cube centered at $\mathbf{u}$. Consider $\mathbf{c}(1)$ :

$$
\begin{aligned}
\mathbf{c}(1) & =O(r+1)-\mathbf{u} \\
& =A I(r+1)+B O(r)-(A \mathbf{v}+B \mathbf{u}) \\
& =A(I(r+1)-\mathbf{v})+B(O(r)-\mathbf{u}) \\
& =A(I(r+1)-\mathbf{v})+B \mathbf{c}(0) .
\end{aligned}
$$

Since $\mathcal{I}$ is a $\delta$-bounded input sequence around $\mathbf{v}$, each $I(r+\Delta t)$ can be denoted as $\mathbf{v}+\delta(r+\Delta t)$ s.t. $\delta(r+\Delta t) \in \mathbb{R}^{n}$ is a vector, and $\|\delta(r+\Delta t)\|_{\infty} \leq \delta$. That is, $\delta(r+\Delta t)=I(r+\Delta t)-\mathbf{v}$.

Note that neither $\mathbf{v}$ nor $\mathbf{u}$ are known. However, the following claim states, that if the nodes follow SS-ItERATIVE, then $\mathbf{c}(\Delta t)$ is bounded and decreases as $\Delta t$ increases.

Claim 1. At round $r+\Delta t$, it holds that $\mathbf{c}(\Delta t)=\sum_{j=0}^{\Delta t-1} B^{j} A \delta(r+\Delta t-j)+B^{\Delta t} \mathbf{c}(0)$.

Proof. Proof by induction. Assume that the claim holds for $\Delta t=k$. Thus, $\mathbf{c}(k)=\sum_{j=0}^{k-1} B^{j} A \delta(r+$ $k-j)+B^{k} \mathbf{c}(0)$. By the update rule in Eq. (5), we have that $O(r+k+1)=A I(r+k+1)+B O(r+k)$. Combining the two equations implies

$$
\begin{aligned}
\mathbf{c}(k+1) & =O(r+k+1)-\mathbf{u} \\
& =A I(r+k+1)+B O(r+k)-(A \mathbf{v}+B \mathbf{u}) \\
& =A(I(r+k+1)-\mathbf{v})+B(O(r+k)-\mathbf{u}) \\
& =A \delta(r+k+1)+B \mathbf{c}(k) \\
& =A \delta(r+k+1)+\sum_{j=0}^{k-1} B^{j+1} A \delta(r+k-j)+B^{k+1} \mathbf{c}(0) \\
& =A \delta(r+k+1)+\sum_{j=1}^{k} B^{j} A \delta(r+k+1-j)+B^{k+1} \mathbf{c}(0) \\
& =\sum_{j=0}^{k} B^{j} A \delta(r+k+1-j)+B^{k+1} \mathbf{c}(0) .
\end{aligned}
$$


Thus, if the claim holds for $\Delta t=k$ it also holds for $\Delta t=k+1$; and we have that the claim holds for all $\Delta t \geq 0$.

Definition 4.1. A matrix $M_{n \times n}$ is diagonally dominant if $\left|M_{i i}\right|>\Sigma_{j \neq i}^{n}\left|M_{i j}\right|$. A matrix $M_{n \times n}$ is normalized diagonally dominant (normalized, for short) if $M$ is diagonally dominant, and $\left|M_{i i}\right| \geq 1$.

Lemma 1. For a normalized diagonally dominant matrix $W$, it holds that $\|A\|_{\infty} \leq 1$ and $\|B\|_{\infty}<$ 1 , where $A, B$ are defined in Eq. (6) and $\|A\|_{\infty} \equiv \max _{x \neq 0} \frac{\|A x\|_{\infty}}{\|x\|_{\infty}}$.

Proof. $A$ is zero except for its main diagonal for which $A_{i, i}=w_{p_{i}, p_{i}}=\frac{1}{w_{i, i}}$. Since $\left|W_{i i}\right| \geq 1$, we have that $\left|A_{i, i}\right| \leq 1$. Thus, it holds that $\|A \mathbf{x}\|_{\infty} \leq\|\mathbf{x}\|_{\infty}$. Furthermore, $\max _{\mathbf{x} \neq 0} \frac{\|A \mathbf{x}\|_{\infty}}{\|\mathbf{x}\|_{\infty}} \leq 1$, i.e., $\|A\|_{\infty} \leq 1$. Regarding $B, B_{i, j}=w_{p_{i}, p_{j}}$ for $i \neq j$ and 0 for $i=j$. Since $W$ is assumed to be normalized diagonally dominant, we have that $\sum_{j \neq i}\left|W_{i, j}\right|<\left|W_{i, i}\right|$, thus $\sum_{j \neq i}\left|w_{p_{i}, p_{j}}\right|<1$. Therefore, $\sum_{j}\left|B_{i, j}\right|=\sum_{j \neq i}\left|w_{p_{i}, p_{j}}\right|<1$ for all $i$. In total, for any $\mathbf{x}$ we have $\|B \mathbf{x}\|_{\infty}<\|\mathbf{x}\|_{\infty}$, leading to $\|B\|_{\infty}<1$.

Theorem 1. Given a normalized diagonally dominant and invertible $W$, there are constants $c_{1}, c_{2}$, where $c_{1}>0$, and $1>c_{2}>0$, such that SS-ITERATIVE $\epsilon$-always converges with $\epsilon(\Delta t, \delta, \mathcal{C})=$ $\delta \cdot c_{1}+\left(c_{2}\right)^{\Delta t} \cdot\|O(r)-\mathbf{u}\|_{\infty}$.

Proof. By Lemma 1 it holds that $\|A\|_{\infty} \leq 1$ and $\|B\|_{\infty}<1$. Consider a $\delta$-bounded input sequence $\mathcal{I}$ around $\mathbf{v}$, and SS-ITERATIVE's run starting from an arbitrary state $O(r)$. We are interested in the behavior of $\|\mathbf{c}(\Delta t)\|_{\infty}$ :

$$
\begin{aligned}
& \|\mathbf{c}(\Delta t)\|_{\infty}=\left\|\sum_{j=0}^{\Delta t-1} B^{j} A \delta(r+\Delta t-j)+B^{\Delta t} \mathbf{c}(0)\right\|_{\infty} \\
& \leq\left\|\sum_{j=0}^{\Delta t-1} B^{j} A \delta(r+\Delta t-j)\right\|_{\infty}+\left\|B^{\Delta t} \mathbf{c}(0)\right\|_{\infty} \\
& \leq \sum_{j=0}^{\Delta t-1}\|B\|_{\infty}^{j}\|A \delta(r+\Delta t-j)\|_{\infty}+\|B\|_{\infty}^{\Delta t}\|\mathbf{c}(0)\|_{\infty} \\
& \leq \delta \cdot\|A\|_{\infty} \sum_{j=0}^{\Delta t-1}\|B\|_{\infty}^{j}+\|B\|_{\infty}^{\Delta t}\|\mathbf{c}(0)\|_{\infty} \\
& =\delta \cdot\|A\|_{\infty} \frac{1-\|B\|_{\infty}^{\Delta t}}{1-\|B\|_{\infty}}+\|B\|_{\infty}^{\Delta t}\|\mathbf{c}(0)\|_{\infty} .
\end{aligned}
$$

For an input sequence $\mathcal{I}$ that is $\delta$-bounded around $\mathbf{v}$, denote by $\mathbf{u}$ the solution to original system of equations $W \mathbf{u}=\mathbf{v}$. By Eq. (7),

$$
\|O(r+\Delta t)-\mathbf{u}\|_{\infty} \leq \delta \cdot\|A\|_{\infty} \frac{1-\|B\|_{\infty}^{\Delta t}}{1-\|B\|_{\infty}}+\|B\|_{\infty}^{\Delta t}\|\mathbf{c}(0)\|_{\infty} .
$$




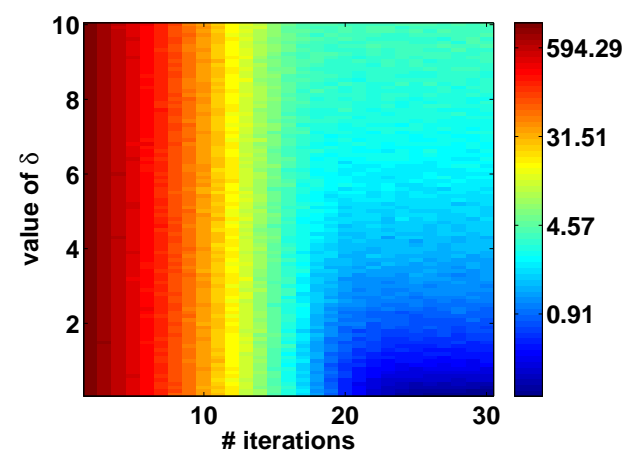

Figure 2: Simulation of a Circle graph.

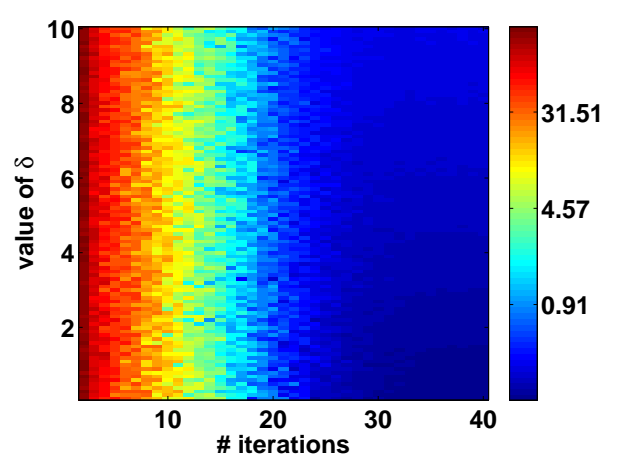

Figure 3: Simulation of a Unit-Disc graph.

Since $\|B\|_{\infty}<1$, we have that $\frac{1-\|B\|_{\infty}^{\Delta t}}{1-\|B\|_{\infty}} \leq \frac{1}{1-\|B\|_{\infty}}$ and by setting $c_{1}=\frac{\|A\|_{\infty}}{1-\|B\|_{\infty}}$ it holds that $\|A\|_{\infty} \frac{1-\|B\|_{\infty}^{\Delta t}}{1-\|B\|_{\infty}} \leq c_{1}$. By setting $c_{2}=\|B\|_{\infty}$ and recalling that $\mathbf{c}(0)=O(r)-\mathbf{u}$ we are done.

Theorem 1 states that SS-ITERATIVE $\epsilon$-always converges. Moreover, the algorithm SS-ITERATIVE is lightweight, as it requires nodes to send only a single value to every neighbor on each round.

\section{Experimental Results}

We have simulated SS-ITERATIVE using two sample topologies of one hundred nodes. Figure 2 depicts a circular topology where each node is connected to its left and right neighbors. Figure 3 shows a random unit disc graph, where nodes are randomly spread on a plane, and each node is connected to the nodes that are within a distance of 1 . The $\mathrm{X}$-axis shows the number of iterations, and the $\mathrm{Y}$-axis shows the value of $\delta$. Area colors in the heatmap depict the average of the following procedure: randomly select a vector $\mathbf{v}$ and a $\delta$-bounded sequence around $\mathbf{v}$, run the simulation for the randomly selected values and return the $L_{\infty}$ distance between the last output vector and $\mathbf{u}$ (calculated as $\mathbf{u}=W^{-1} \mathbf{v}$ ). The heatmap uses a $\log \log$ scale. Both graphs clearly show that as $\delta$ decreases and the number of iterations increases, the output of SS-ITERATIVE converges to be bounded by a small cube around $\mathbf{u}$.

Note that the unit disc weighted topology matrix is characterized by $\|A\|_{\infty}=0.02,\|B\|_{\infty}=0.97$ while the circle graph is characterized by $\|A\|_{\infty}=0.33,\|B\|_{\infty}=0.66$. As expected, using unit disc topology requires a larger number of iterations for convergence (depends on $\|B\|_{\infty}$ ). In addition, in the unit disc topology the value of $\delta$ has a lesser affect on the convergence, due to the value of $\|A\|_{\infty}$, which affects the minimal radius around the output. Since $\|A\|_{\infty}$ is smaller in the unit disc topology, increasing $\delta$ does not significantly affect the convergence.

\section{Extension to the asynchronous model}

Our second novel contribution is in extending our model to support asynchronous communications. In a large sensor network, it is not reasonable to assume that the sensor operate in synchronous rounds. Furthermore, as known from the linear iterative algorithms literature, when working in asynchronous rounds, the algorithms usually converge faster. 
When considering the asynchronous model, it is more convenient to discuss shared-memory as means of communication. ${ }^{3}$ Thus, assume that for each directed edges between $p_{i}, p_{j}$ there is a read-write register $R_{p_{i}, p_{j}}$ that is written by $p_{i}$ and read by $p_{j}$.

An asynchronous run is an infinite sequence of of configurations $\mathcal{C}_{0} \rightarrow \mathcal{C}_{1} \rightarrow \ldots$ such that some process $p$ performs an atomic step between configuration $\mathcal{C}_{i}$ and $\mathcal{C}_{i+1}$. An atomic step consists of reading or writing from a single register. Notice that in the current model a configuration consists of all of the registers and of the local variables at the different nodes.

In this section we again prove that starting from an arbitrary configuration, when the inputs are bounded, the outputs are bounded as well. We consider each configuration $\mathcal{C}_{r}$ to be assigned a vector input $I(r)$ such that if node $p_{i}$ reads the input when performing an atomic step on $\mathcal{C}_{r}$ it reads the value of $I_{p_{i}}(r)$. Equivalently, the output vector of configuration $\mathcal{C}_{r}$ is $O(r)$.

Figure 4 outlines ASYNC-SS-ITERATIVE which is a direct translation of SS-ITERATIVE to the shared-memory model.

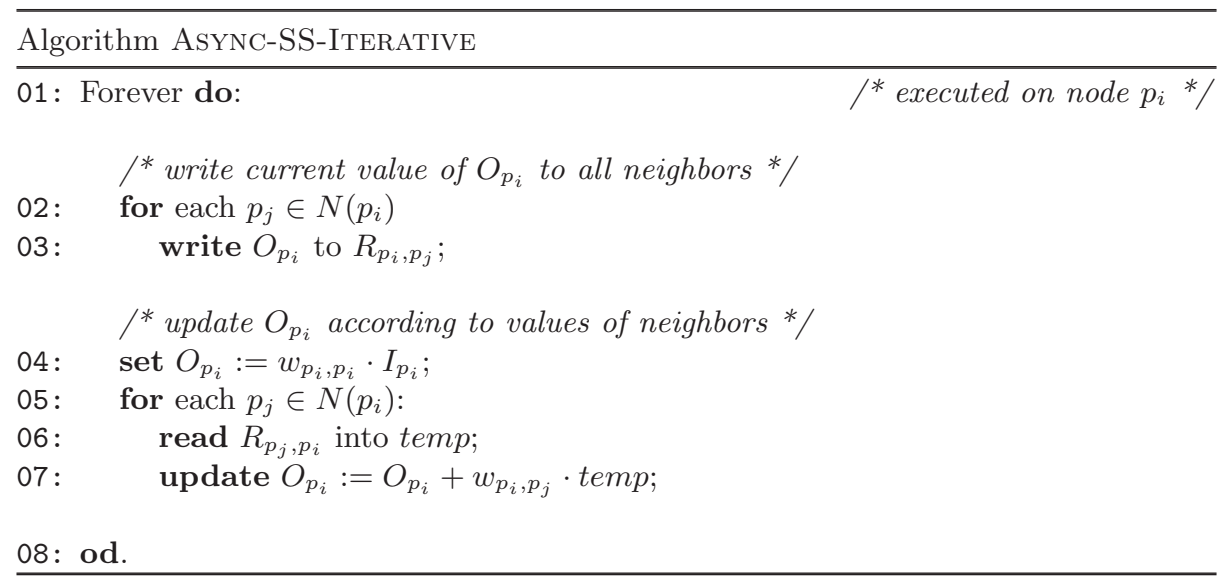

Figure 4: A self-stabilizing iterative algorithm for asynchronous networks.

ASYNC-SS-ITERATIVE consists of two phases: in the first, the previous value of $O_{p_{i}}$ is written to all its neighbors. In the second phase $p_{i}$ calculates its new value of $O_{p_{i}}$ by reading the registers of all its neighbors.

We consider only "fair" runs, in which each node performs an atomic step infinitely many times. Thus, each node performs both phases infinitely many times. A round is defined to be the shorted prefix of a run such that each node has performed all steps (a)-(d) in the algorithm. We number each atonic step and each round. The first round consists of many atomic steps.

We model a fair run as follows. Each node $p_{i}$ performs infinitely many atomic steps, and participates in infinitely many rounds. Notice that the registers $p_{i}$ reads in round $k+1$ have all been written to, no earlier than during round $k$. Since a round consists of each node performing all the steps in the algorithm, each node $p_{i}$ manages to read all of its neighboring registers and write to all of its neighboring registers every round. Thus, there is some atomic step $r$ (during round

\footnotetext{
${ }^{3}$ In [7] it is shown how to convert an algorithm based on shared-memory to a message-passing algorithm with links of bounded capacity.
} 
$k+1)$ such that:

$$
O_{p_{i}}(r)=w_{p_{i}, p_{i}} \cdot I_{p_{i}}\left(r^{\prime}\right)+\sum_{j \neq i} w_{p_{i}, p_{j}} \cdot O_{p_{j}}\left(r_{j}^{\prime}\right) .
$$

such that $r^{\prime}, r_{j}^{\prime}$ (for the for all $p_{j} \neq p_{i}$ ) are smaller than $r$ and are from at least round $k$.

Let $\mathbf{u}$ be such that $\mathbf{u}=A \mathbf{v}+B \mathbf{u}$, and let the inputs be from a $\delta$-bounded input sequence around $\mathbf{v}$. Denote by $\mathbf{c}(r)=O(r)-\mathbf{u}$. Notice that if $p_{i}$ did not perform the $r$ th atomic step then $O_{p_{i}}(r)=O_{p_{i}}(r-1)$ and therefore $\mathbf{c}_{p_{i}}(r)=\mathbf{c}_{p_{i}}(r-1)$. Consider the value of $\mathbf{c}_{p_{i}}(r)$ when $p_{i}$ did perform the $r$ th atomic step (during round $k+1$ ).

$$
\begin{aligned}
\mathbf{c}_{p_{i}}(r) & =O_{p_{i}}(r)-\mathbf{u}_{p_{i}} \\
& =w_{p_{i}, p_{i}} \cdot I_{p_{i}}\left(r^{\prime}\right)+\sum_{j \neq i} w_{p_{i}, p_{j}} \cdot O_{p_{j}}\left(r_{j}^{\prime}\right)-w_{p_{i}, p_{i}} \cdot \mathbf{v}_{p_{i}}-\sum_{j \neq i} w_{p_{i}, p_{j}} \cdot \mathbf{u}_{p_{i}} \\
& =w_{p_{i}, p_{i}} \cdot\left(I_{p_{i}}\left(r^{\prime}\right)-\mathbf{v}_{p_{i}}\right)+\sum_{j \neq i} w_{p_{i}, p_{j}} \cdot\left(O_{p_{j}}\left(r_{j}^{\prime}\right)-\mathbf{u}_{p_{i}}\right) \\
& =w_{p_{i}, p_{i}} \cdot\left(I_{p_{i}}\left(r^{\prime}\right)-\mathbf{v}_{p_{i}}\right)+\sum_{j \neq i} w_{p_{i}, p_{j}} \cdot \mathbf{c}_{p_{j}}\left(r_{j}^{\prime}\right)
\end{aligned}
$$

where $r^{\prime}$ and the different $r_{j}^{\prime}$ are smaller than $r$ and are all from round $k$ or round $k+1$.

By using Lemma 1 we get:

$$
\begin{aligned}
\left|\mathbf{c}_{p_{i}}(r)\right| & \leq\left|w_{p_{i}, p_{i}} \cdot\left(I_{p_{i}}\left(r^{\prime}\right)-\mathbf{v}_{p_{i}}\right)\right|+\max _{p_{j}}\left|c_{p_{j}}\left(r_{j}^{\prime}\right)\right| \sum_{j \neq i}\left|w_{p_{i}, p_{j}}\right| \\
& \leq\left|w_{p_{i}, p_{i}} \cdot\left(I_{p_{i}}\left(r^{\prime}\right)-\mathbf{v}_{p_{i}}\right)\right|+\|\left. B\right|_{\infty}\left|\mathbf{c}_{p_{\max }}\left(r_{\max }\right)\right| \\
& \leq \delta+\|B\|_{\infty}\left|\mathbf{c}_{p_{\max }}\left(r_{\max }\right)\right|
\end{aligned}
$$

for some $p_{\max }$ and $r_{\max } \leq r$ that is from round $k$ or $k+1$.

Therefore, for any $p_{i}$ during round $k+1$ there is a list of length $\ell \geq k$ of nodes $p_{1}, p_{2}, \ldots, p_{\ell}$ and a sequence of length $\ell$ of atomic steps $r_{1}>r_{2}>\cdots>r_{\ell}=0$, such that

$$
\begin{aligned}
\left|\mathbf{c}_{p_{i}}(r)\right| & \leq \delta+\|B\|_{\infty}\left|\mathbf{c}_{p_{1}}\left(r_{1}\right)\right| \\
& \leq \delta+\|B\|_{\infty}\left(\delta+\|B\|_{\infty}\left|\mathbf{c}_{p_{2}}\left(r_{2}\right)\right|\right) \\
& =\delta \cdot\left(1+\|B\|_{\infty}\right)+\|B\|_{\infty}^{2}\left|\mathbf{c}_{p_{2}}\left(r_{2}\right)\right| \\
& \leq \delta \cdot \sum_{z=0}^{\ell-1}\|B\|_{\infty}^{z}+\|B\|_{\infty}^{\ell}\left|\mathbf{c}_{p_{\ell}}\left(r_{\ell}\right)\right| \\
& =\delta \cdot \frac{1-\|B\|_{\infty}^{\ell}}{1-\|B\|_{\infty}}+\|B\|_{\infty}^{\ell}\left|\mathbf{c}_{p_{\ell}}(0)\right| .
\end{aligned}
$$

Denote by $z_{1} \triangleq \max _{i}\left|\mathbf{c}_{p_{i}}(0)\right|, z_{2} \triangleq \frac{1}{1-\|B\|_{\infty}}$, and $z_{3} \triangleq\|B\|_{\infty}$. We have that for node $p_{i}$ performing the $r$ th atomic step during round $k$ it holds that $\left|\mathbf{c}_{p_{i}}(r)\right| \leq \delta \cdot z_{2}+z_{3}^{\ell} \cdot z_{1} \leq \delta \cdot z_{2}+z_{3}^{k} \cdot z_{1}$. In fair runs, there are infinitely many rounds $k$, thus, as $r$ goes to infinity, we have that $\|O(r)\|_{\infty}$ is bounded by a cube of length $\delta \cdot z_{2}$ around $\mathbf{v}$. 


\section{Discussion}

We have shown that the algorithm SS-ITERATIVE is a modification of the Jacobi iterative method to solve a set of equations $A \mathbf{x}=\mathbf{b}$, where $A$ is given and $\mathbf{b}$ is dynamically changing but bounded. Moreover, Theorem 1 is a generalization of previous analysis of Jacobi's convergence. Our motivation for SS-ITERATIVE originates from the sensor calibration problem where sensors need to calibrate their noisy readings. Unlike previous approaches to this problem, we assume a dynamic system with an infinite execution of the algorithm. In this setting the readings of the sensors continuously change. Under the assumption that the readings' changes are bounded, we have shown that the calibrated output is bounded as well.

Further application for SS-ITERATIVE can be found in any setting where it is desired to solve $A \mathbf{x}=\mathbf{b}$ in a converging and self-stabilizing manner, while $A$ is given, and $\mathbf{b}$ may change slightly from one round to the next. Notice that the analysis given in Section 4 holds in such a system.

As noted in Remark 3.2 the matrix $A$ is "part of the code". An optional alternative to the current solution is to compute $A^{-1}$ (the inverted matrix of $A$ ) beforehand and include it "as part of the code". Thus, each node could locally solve $\mathbf{x}=A^{-1} \mathbf{b}$, and it can be shown that $\mathbf{x}$ will be bounded (as long as $\mathbf{b}$ is bounded). The main problem with such a solution is the connectivity requirements it incurs. In our solution, scalar values are sent in the network only between direct neighbors. The matrix $W$ represents a weighted adjacency graph. Once inverted, the matrix $W^{-1}$ might not be sparse. A non-zero entry $w_{i j}^{-1} \in W^{-1}$ means that node $p_{i}$ needs to communicate with node $p_{j}$. This extra communication might cause the algorithm to lose its self-stabilizing properties, as non-neighboring nodes would require a self-stabilizing overlay network for their communication.

The assumption of a predefined $A$ is suitable for static networks in which the communication graph is predetermined. For dynamic networks, it would be interesting to adjust SS-ITERATIVE to discover the connectivity of the network, inferring the optimal weights dynamically. We assume that after the weights are calculated, the topology of the sensor network remains stable, thus the convergence analysis of Section 4 should hold.

\subsection{Relation to Perturbation Theory}

A large amount of research was focus at the problem of solving $A \mathbf{x}=\mathbf{b}$ when $A$ and $\mathbf{b}$ are not exactly known. That is, let $\hat{A}=A+\delta A$ and $\hat{\mathbf{b}}=\mathbf{b}+\delta \mathbf{b}$, and consider the equation $\hat{A} \hat{\mathbf{x}}=\hat{\mathbf{b}}$; what can be said about $\mathrm{x}$ in relation to $\hat{\mathbf{x}}$ ?

Our setting is "easier" in one sense, and "harder" in a different sense. In our setting $A$ is known, i.e., $\delta A=0$. However, $\hat{\mathbf{b}}$ is not well defined. That is, the input vector - which is described by $\hat{\mathbf{b}}$ - changes over time. When solving $\hat{A} \hat{\mathbf{x}}=\hat{\mathbf{b}}$ it is assumed that there is some $\mathbf{b}$ that is constant but it was measured with an error. In our case, $\mathbf{b}$ is not constant as it changes over time, while it represents the measurement correctly.

As a future research, tt would be interesting to consider the implications of adding inaccuracy to the measurements. The vast body of knowledge regarding perturbation theory would definitely aid in this extension to our model.

\subsection{Relation to convex optimization}

Many practical optimization problems are given in the quadratic form $f(x)=1 / 2 \mathbf{x} A \mathbf{x}-\mathbf{b}^{T} \mathbf{x}$, where the task is to compute $\min _{\mathbf{x}} f(\mathbf{x})$ distributively over a communication network. A survey 
showing several applications can be found in [3]. Example applications are monitoring, distributed computation of trust and ranking of nodes and data items.

A standard way for solving $\min _{\mathbf{x}} f(\mathbf{x})$ is by computing the derivative and comparing it to zero to get the global optimum. When the matrix $A$ is symmetric, $f^{\prime}(\mathbf{x})=A \mathbf{x}-\mathbf{b}=0$, and we get a linear system of equations $A \mathbf{x}=\mathbf{b}$. In other words, the convex optimization problem is reduced into a solution of linear system of equations.

Interior point methods $[4, \S 11]$ solve linear programming problems by applying Newton method iteratively. Each computation of the Newton step involves a solution of a linear systems of equations. An area of future work is to examine the applications of our self-stabilizing algorithm to these methods. The difficulties arise from the fact that the matrix A needs to be recomputed between iterations, so nodes need to be synchronized and aware to the current iteration taking place.

\section{Acknowledgements}

Ezra N. Hoch would like to thank Golan Pundak for assisting with the simulations.

\section{References}

[1] R. M. Bell and Y. Koren. Scalable collaborative filtering with jointly derived neighborhood interpolation weights. In IEEE International Conference on Data Mining (ICDM'07), 2007.

[2] D. P. Bertsekas and J. N. Tsitsiklis. Parallel and Distributed Calculation. Numerical Methods. Prentice Hall, 1989.

[3] D. Bickson and D. Malkhi. A unifying framework for rating users and data items in peerto-peer and social networks. In Peer-to-Peer Networking and Applications (PPNA) Journal, Springer-Verlag, 2008.

[4] Stephen Boyd and Lieven Vandenberghe. Convex Optimization. Cambridge University Press, March 2004.

[5] L. Davidovitch, S. Dolev, and S. Rajsbaum. Stability of multivalued continuous consensus. SIAM Journal on Computing, 37(4):1057-1076, 2007.

[6] D. Dolev and E. N. Hoch. Ocd: Obsessive consensus disorder (or repetitive consensus). In Proc. of the 27st Int. Symposium on Principles of Distributed Computing (PODC'08), Tornoto, Canada, Aug. 2008.

[7] S. Dolev. Self-Stabilization. The MIT Press, 2000.

[8] S. Dolev and S. Rajsbaum. Stability of long-lived consensus. J. Comput. Syst. Sci., 67(1):2645, 2003.

[9] Jun Fang and Hongbin Li. Distributed event-region detection in wireless sensor networks. In EURASIP J. Adv. Signal Process, volume 2008, pages 1-10, New York, NY, United States, January 2008. Hindawi Publishing Corp. 
[10] Koen Langendoen and Niels Reijers. Distributed localization in wireless sensor networks: a quantitative comparison. In Comput. Networks, volume 43, pages 499-518, New York, NY, USA, November 2003. Elsevier North-Holland, Inc.

[11] Keith Marzullo. Tolerating failures of continuous-valued sensors. ACM Trans. Comput. Syst., 8(4):284-304, 1990.

[12] R. Olfati-Saber. Distributed kalman filtering for sensor networks. In Proc. of the 46th IEEE Conference on Decision and Control, December 2007. 